\title{
Some Observations upon "Realistic" Trajectories in Bohmian Quantum Mechanics*
}

\author{
María C. BosCÁ
}

Received: 4.6.2008

Final version: 1.1 .2012

BIBLID [0495-4548 (2013) 28: 76; pp. 45-60]

ABSTRACT: Experimental situations in which we observe quantum effects that deviate from the intuitive expectations of the classical world call for an interdisciplinary discussion, and one fundamental issue to be considered is the compatibility between the description of phenomena and the assumption of an objective reality. This paper discusses the ontological interpretation of Bohmian quantum mechanics, focusing on the use of the term "trajectory" and the difficulties associated with its connection to a "real" (objective) trajectory. My conclusion is that the intended realistic interpretation of Bohmian trajectories is highly questionable.

Keywords: quantum mechanics; Bohmian quantum mechanics; realistic trajectory; quantum position.

RESUMEN: Los contextos experimentales que permiten la observación de efectos cuánticos contrarios a los esperables según la intuición clásica son especialmente adecuados para una discusión multidisciplinar, en particular sobre la compatibilidad entre la descripción fenomenológica y una pretendida realidad subyacente. Este artículo discute la interpretación ontológica de la mecánica cuántica en la interpretación de Bohm, centrándose principalmente en su uso del término "trayectoria" y las dificultades inherentes a su conexión con un concepto "real” (objetivo) de la misma. La conclusión es que la pretendida interpretación realista de las trayectorias bohmianas es muy cuestionable.

Palabras clave: mecánica cuántica; interpretación de Bohm; trayectoria bohmiana; trayectoria realista; posición.

\section{Introduction}

The ontological status of the theoretical entities, introduced to achieve an explanation of phenomena, constitutes a crucial debate between realists and instrumentalists. Ontology occupies a central place in any realistic concept of science, and basic ontology, the reference to what exists and happens in the world, encapsulates the explanation for the success of theories. To instrumentalists, the question of the ontological significance of a mathematical formalism, provided that it is empirically correct, is irrelevant.

It has been argued that a clear ontological commitment is possible only when a scientific discipline becomes mature, but modern quantum physics continues to reject such a commitment. Newtonian mechanics establishes a clear basic ontology of material bodies as individual objects and provides for the encoding of dynamic processes on the basis of precise configurations of particle positions and velocities and their related symmetries. This ontological foundation collapsed in orthodox quantum physics

\footnotetext{
* The research for this paper was supported by the Spanish Ministry of Science and Innovation (project FFI2008-06418-C03-02). The author also thanks two anonymous reviewers for their helpful comments.
} 
however and, in view of its enormous empirical success, the groundlessness of assigning real nature to quantum entities seemed to be vindicated ${ }^{1}$.

By reviewing different experiments, I discuss here the semantic and ontological commitments related to some of the theoretical terms that appear in such experimental contexts and whether it is possible or not to establish some sense of continuity with regard to the corresponding classical terms. Specifically, this paper elucidates the question of whether the particle trajectories in the Bohmian interpretation of quantum mechanics can be properly called "realistic" in the two following senses: a) each particle occupies a clear, objective position, irrespective of whether it is observed or not; b) in the cases where a macroscopic picture emerges (a trace of bubbles is generated by a particle, for instance), the corresponding Bohmian variable is compatible with the intuitive classical picture (the particle moves following a path consistent with that marked by the bubbles).

\section{Trajectory in orthodox quantum mechanics}

The basis of the objective world-view of classical (particle) physics is the assumption that individual objects always possess well defined properties and traverse well defined spatio-temporal trajectories. But, as Heisenberg has commented (Heisenberg 1985, 169), after suddenly remembering Einstein's statement "it is the theory which decides what we can observe", he realized that the path of the electron in the cloud chamber was not really observed:

We have always said so glibly that the path of the electron in the cloud chamber could be observed. But perhaps what we really observed was something much less. Perhaps we merely saw a series of discrete and ill-defined spots through which the electron has passed. In fact, all we do see in the cloud chamber are individual water droplets which must certainly be much larger than the electron. The right question should therefore be: Can quantum mechanics represent the fact that an electron finds itself approximately in a given place and that it moves approximately with a given velocity, and can we make these approximations so close that they do not cause experimental difficulties? (Heisenberg 1985, 169)

Consequently, in 1927 Heisenberg concluded that "no interpretation of quantum mechanics is possible which uses ordinary kinematical and mechanical concepts", and thus stated:

Quantum mechanics arose exactly out of the attempt to break with all ordinary kinematic concepts and to put in their place relations between concrete and experimentally determinable numbers.

[...] In quantum mechanics, however, the relation $\mathrm{pq}-\mathrm{qp}=-\mathrm{ih} / 2 \pi$ between mass, position, and velocity is believed to hold. Therefore we have good reasons to become suspicious every time uncritical use is made of the words "position" and "velocity". When one admits that discontinuities are somehow typical of processes that take place in small regions and in short times, then a contradiction between the concepts of "position" and "velocity" is quite plausible. (Heisenberg $1983,62-63)$

\footnotetext{
${ }^{1}$ Bell's theorem (Bell 1964) states that no local-hidden-variable theory can predict the same results as those given by standard quantum mechanics in certain experimental situations. The experiments carried out until now, blocking successive loopholes, agree with quantum predictions.
} 
In the same paper he remarked:

When one wants to be clear about what is to be understood by the words "position of the object", for example of the electron (relative to a given frame of reference), then one must specify definite experiments with whose help one plans to measure the "position of the electron"; otherwise this word has no meaning.

[...] We turn now to the concept of "path of the electron". By path we understand a series of points in space (in a given reference system) which the electron takes as "positions" one after the other. As we already know what is to be understood by "position at a definite time", no new difficulties occur here. Nevertheless, it is easy to recognize that, for example, the frequently used expression, the " $1 \mathrm{~s}$ orbit of the electron in the hydrogen atom," from our point of view makes no sense. In order to measure this 1s "path" we have to illuminate the atom with light whose wavelength is considerably shorter than $10^{-8} \mathrm{~cm}$. A single photon of such light, however, is enough to eject the electron completely from its "path" (so that only a single point of such a path can be defined). Therefore, the word "path" in this context has no definable meaning. This conclusion can already be deduced, without any knowledge of the recent theories, simply from experimental possibilities.

[...] All concepts that can be used in classical theory for the description of a mechanical system can also be precisely defined for atomic processes in analogy to the classical concepts.

[...] I believe that one can fruitfully formulate the origin of the classical "orbit" in this way: the "orbit" comes into being only when we observe it. (Heisenberg 1983, 64-73)

Thus, as is well known, the formulation of the indeterminacy principle ruled the concept of spatio-temporal trajectory out of quantum theory.

Nowadays, new experiments have shown how far the above words of Heisenberg conform to the observed phenomena, and how we must be very careful with the use, not only of the theoretical term "spatio-temporal trajectory", but also of the common notion of a real, objective path travelled along. To show this, I shall refer to the recent experimental realization (Jacques et al. 2007) of Wheeler's gedanken two-slit delayedchoice experiment (Wheeler 1978), inspired by some passages in the Einstein-Bohr dialogue, in which the choice between measuring a single entity's particle-like properties (only one path) and wave-like properties (both paths) is made after the quantum entity has gone through the slits.

The quantum two-slit experiment is, in fact, merely the quantum version of Young's classical one undertaken during the first decade of the nineteenth century, in which faint light passes through two slits to produce interference fringes, observed on a screen located at some distance. The quantum version interferes not only with light but also with material objects such as electrons, for example. The theoretical prediction foresees the appearance of interference fringes (for a suitable set of parameters) and was confirmed experimentally first in 1961 (Jönsson 1961) and subsequently with electrons sent one at a time through the device (Merli et al. 1976; Tonomura et al. 1989). In recent years the experiment has been undertaken even with complex molecules such as fullerenes ${ }^{2}$ (Zeilinger 1999) and attempts are due to be made with certain viruses.

It is well-known that the classical treatment of the experiment with material particles is unable to predict the confirmed observation of interference fringes. If we imag-

${ }^{2}$ A $\mathrm{C}_{60}$ system: 60 nuclei and 360 electrons. 
ine marbles that are successively being fired against a plane with two slits of a suitable width, what is classically predicted for the distribution of impacts on the rear screen are simply two bands of the same size. In the quantum case ${ }^{3}$, whenever the two paths are indistinguishable, an additional interference term emerges between the two state vectors corresponding to situations with only one slit open, which results in a density of impacts on the screen that displays alternating bands of areas where the impacts are concentrated and areas where only a few are produced: an interference pattern.

The interferential figure emerges gradually as the successive records of impacts are being produced on the screen. Where each individual impact will occur is unpredictable; after each one occurs exactly the same will apply to where the following one will be recorded; finally, for each global accomplishment of the experiment, the overall pattern of individual impacts is different and impossible to predict (lack of determinism). If the experiment includes a device for detecting (with certainty) which slit each particle passes through it is impossible to observe the interference fringes, and the distribution of impacts is the classically expected one. The theory generates the correct prediction again; in this case, the interference term between the two possibilities is null.

The delayed-choice version is a modified version of this two-slit experiment; the idea, in brief, is to decide whether to determine or not which slit has been traversed once the experiment is already underway. The new experiment (Jacques et al. 2007) replaces the traditional device by a two-way Mach-Zehnder interferometer ${ }^{4}$, which is entered by single-photon light pulses. The setting consists of two beam-splitters, two mirrors and two detectors. Pulses of linearly polarized single photons are sent by a first polarization beam-splitter through an interferometer (length $48 \mathrm{~m}$ ) with two possible spatially separated paths; the mirrors allow the recombination of beams in a zone where they can either find or not the second beam-splitter; behind it, two detectors are placed.

The experiment is, in principle, fully equivalent to the usual one: if the second beam-splitter is absent, the presence of the two detectors renders the two paths distinguishable, no interference is observed and equal probabilities are measured on the two detectors, it being impossible to predict which one will click every time. But, if we add the second beam-splitter, the recombination of the two beams renders the two paths indistinguishable and, by varying the relative phase between them, the result is a counting rate modulated by a trigonometric function, which corresponds to the traditional observation of interference fringes.

Since the insertion or not of the second beam-splitter occurs randomly in a region relativistically separated from the entry of each photon into the device, the quantum orthodox description of the results is incompatible with any representation or verbal-

${ }^{3}$ In fact, all cases are quantum. What happens is that it is impossible to observe interference with macroscopic objects: the maxima and minima of the total intensity would be so close that we could not distinguish between them with detectors of finite size and only observe their average, which coincides with the sum of the intensities for each slit.

${ }^{4}$ The Mach-Zehnder interferometer is a particularly simple device for demonstrating interference by division of amplitude (see e.g. Zetie et al. 2000).

Theoria 76 (2013): 45-60 
ized image of the phenomena prior to the reading of the records in the output ports. Only after the experiment has been carried out, if a setting without the second beamsplitter was chosen, then, by resorting to Bohr's complementarity, we could say that it corresponded to the exhibition of a corpuscular nature, and "the photon followed only one of the two paths". But if, on the contrary, the experiment was finally carried out with the two beam-splitters in place, then we can say that we have observed wave properties, and "the photon followed both paths".

It is evident that the latter choice of experimental setting does not determine the choice made by the entity at some earlier time, as is sometimes maintained, but that it is meaningless to talk about phenomena which are not actually registered as being "real" phenomena. The dramatic epistemological consequences of this kind of experiment were described using the metaphor of a "great smoky dragon" (Kennedy 1985, 151), with only two parts sharply defined: the tail, where the entity enters the apparatus, and the mouth, where it is detected. All the rest is a fog of uncertainty: in between "we have no right" to speak about what is present, as "no elementary phenomenon is a phenomenon until it is a registered (observed) phenomenon" (Wheeler 1983).

The orthodox interpretation can be affirmed like an interpretation of minima, which simply states correlations between the initial preparation of a physical system, represented by a density operator, with the full sequence of possible outcomes, through the corresponding probabilities; in addition to that, it assumes a fundamental indeterminism and disqualifies most of the questions on an underlying reality to the phenomena like metaphysics. The wave function is assumed like a mere mathematical tool to evaluate those probabilities, discarding any additional interpretation. The theory predicts all the experimental results to date correctly, which, from an instrumental point of view, is all that is required.

In a quantum experiment, we can usually distinguish three phases. The first corresponds to the preparation of the experiment, and in a delayed-choice setting, it temporally coincides with the second, the phase of proper realization of the experiment. In this second phase, there is no distinction between the observed and the experimental apparatus used, which are correlationated: the border between the observed and the observer has become completely blurred, and it has lost the distinction of subject/object, essential to appeal to an objective reality. In addition to that, at this stage there is no experimental narration, that is to say, neither a representation nor visualization is provided for what is happening: the black box is the most correct model, if any is required. The third and final phase consists in the reading of registers on the counters.

Next, after the experiment has finished, it is possible to build a narrative description of the realized experience, although it does not add any new knowledge: all the possible information is already contained in the measured records, so that it is a completely dispensable step in a purely instrumentalist option. The narration implies that a mental model for what has happened is constructed, a model that usually (Bohr believed it was inevitably), in order to be unambiguous, has to use classical concepts, the ones directly related to our quotidian intuitions and that are forged for beings who live 
in the macroscopic world. For instance: the results of an experiment like the quantum two-slit are frequently narrated in a classical language, including expressions like "the photon went to the first slit", but it only expresses what we may "say" about what (classically) seems to appear to have happened, once the whole experiment has been carried out. And, in a delayed choice experiment, it means neither have we "created" a past fact, nor that, if we had been looking at an earlier time, we would have found the photon travelling along the referred path. The notion of objective path travelled along has completely faded away, and the suggested image of each photon travelling along a given path is a purely linguistic resource, connected to a classical mental representation incompatible with the quantum formalism.

Once this meaning 5 is accepted, it is clear that we are compelled to abandon any plausible idea of attaching an ontological commitment to orthodox quantum physics: there is not a new quantum ontology for the microscopic level, and classical macroscopic ontology is referred to in order to communicate the results of experiments. Suggestively, the narrative of the events elaborated by appealing to the classical ontology is not possible for all experimental contexts; when it is possible, it provides the fundamental link between the objective description of nature realized by classical physics (which is possible only in the scope of the macroscopic world) and some quantum phenomenology.

\section{Trajectories in Bohmian quantum mechanics}

It is well known that some of the founding fathers of quantum mechanics, mainly Schrödinger and Einstein, were deeply dissatisfied with the new theory, as "no reasonable definition of reality could be expected to permit this" (Einstein et al. 1935, 780). In 1952, David Bohm, following some previous ideas of de Broglie (Broglie 1927), presented a new formulation, a fully deterministic and explicitly non-local theory (Bohm 1952). The new interpretation (Bohm et al. 1987; Bohm and Hiley 1993), which was presented in close analogy to the Hamilton-Jacobi formulation of classical mechanics, represented mathematically a system consisting of two elements: points in physical space (particles) and the wave function, or pilot wave, in configuration space, which guides their movements. It emphasises a state-dependent potential that changes instantaneously throughout configuration space whenever the wave function changes, a mechanism that is responsible for non-local correlations ${ }^{6}$.

5 "There is no quantum world. There is only an abstract quantum physical description. It is wrong to think that the task of physics is to find out how nature is. Physics concerns what we can say about nature." (Bohr, cit. in Petersen 1985, 305); "These facts not only set a limit to the extent of the information obtainable by measurement, but they also set a limit to the meaning which we may attribute to such information." (Bohr 1934, 18)

${ }^{6}$ Dürr et al. presented (1992a) another version which, in their opinion, makes it clearer that Bohmian mechanics is a new, distinctly non-Newtonian mechanics. For instance, it turns out that because of the effects of its wave function on the evolution of its position, the trajectory of a single particle in free space is not necessarily a simple straight line; their version hides the quantum potential, which reappears when the transformation to Bohm's original version is made. 
Bohm and Hiley called Bohmian mechanics an "ontological interpretation of quantum theory" because it is grounded in ontology rather than in epistemology, contrary to the orthodox formulation. As it has been stated (Goldstein 1996; Dürr et al. 1992b, 1996), this formulation arises when we assume an ontology of particles to whose motion the Schrödinger equation, the common part of almost all interpretations of quantum theory, is relevant. It has been said that "Bohmian Mechanics is the theory that emerges when we indeed insist that 'particles' mean particles" (Dürr et al. 1996, 21), a not very clear statement in which we suppose that the term "particles" has the meaning that they stated to be 'usual': "point-like entities whose most important feature is their position in space" (that is to say, are described by their position, which is an objective property, existing regardless of whether or not it is observed), and possess mass, momentum and other properties, most of them contextual. In any case, it should be made clear that, as Bohmian mechanics is by no means Newtonian7, "particle" cannot just mean "classical particle", in spite of the fact that its position is claimed to be objective. Furthermore, it must be emphasized that the meaning of theoretical terms is determined by the corresponding theory, and in Bohmian mechanics only the position of particles is always considered to be an intrinsic property, whilst most of the remaining observable quantities, such as momentum ${ }^{8}$, spin, energy and so on, obtain meaning only through their association to specific experiments, this is, they are contextual (Dürr et al. 1996; Daumer et al. 1996), just as it is true for them in orthodox quantum theory. Consequently, an ideal position measurement will, supposedly, reveal the objective position of the particle, but the results for other properties would be contextualized, thus, will depend upon the used experimental set-up.

Therefore, as has been repeatedly emphasized, Bohmian mechanics does not represent a return to the classical paradigm, but, like orthodox quantum mechanics, implies a radical challenge to it. In opposition to the orthodox theory, to which it is empirically equivalent (to non-relativistic theory), it assumes an objective quantum reality, made of particles, with well-defined positions and following a trajectory in physical space, together with a wave-field.

In a two-slit experiment, the particle follows a well-defined trajectory, the mathematical one-parameter solution of Bohm's modification of Hamilton-Jacobi's equation, and goes through one or other of the slits, whilst the wave goes through both. The theory produces the set of trajectories that is required to obtain the usual interference pattern; each individual particle passes through one single slit, which can be de-

\footnotetext{
${ }^{7}$ For instance, if Newtonian mechanics is a second-order physics, where accelerations and forces play a fundamental role, Bohmian mechanics is a first-order physics, in which it is the velocities, the rates of change of position - which are not independent of position, as they are classically-, that are fundamental (Dürr et al. 1996; 1992b, 8); the fundamental question of non-separability deserves special mention.

${ }^{8}$ Since each particle has a definite trajectory in the Bohmian formalism, it would be possible to speak of a non-contextual momentum, but then this, let us say, "actual" momentum, is not a generally observable property, that is, differs from the measured momentum (see e.g. Myrvold 2003). In fact, in Bohmian theory the measured momentum is not the same as the mass-times-particle-velocity momentum: the first is conserved but not the second.
} 
duced from its final position: they are retrodicted trajectories (for a set of figures showing these trajectories see e.g. Philippidis et al. 1979).

In 1992 Englert et al. claimed to have shown, in the context of a thought experiment, that-at least in certain situations-Bohmian trajectories differ from the socalled "observed" trajectories, that is, the paths the quantum system appears to have followed when a measurement result has been established (Englert et al. 1992). Consequently, they concluded that Bohm's trajectories are not real particle trajectories but "surreal" ones. The corresponding experimental arrangement was ultimately, yet again, a two-slit delayed-choice experiment, similar to the standard but this time with crossing paths, a kind of situation that was discussed by Bell, showing that symmetry ensures that, in Bohmian mechanics, if at any given time the particle is above (below) the symmetry plane between the two slits, then it will always remain above (below) that plane (Bell 1980, 157). Their which-way device was supplemented with electromagnetic cavities ("micromaser Welcher-Weg detectors"), which act as one-bit whichway detectors capable of recording through which slit the atom went without disturbing the atom's center-of-mass motion, via the deposit of a photon in the cavity as a direct consequence of the dynamically local interaction of the atom with the cavity field. They found that there were events in which the track recorded by the which-way detectors was always different from the corresponding Bohm trajectory:

The advanced techniques of modern quantum optics enable the experimenter to build one-bit detectors sensitive to the passage of a single atom. [...] We shall symbolize the one bit-recording as a transition from "no" to "yes". It is important to realize that this transition happens with virtual certainty and that the atom's center of mass wave function is not altered noticeably in the process.

[...] There will be events when the atom goes through the upper detector and therefore through the upper slit and then hits the lower half of the screen, so that the corresponding Bohm trajectory goes though the lower slit. In other words: the Bohm trajectory is here macroscopically at variance with the actual, that is: observed track. Tersely: Bohm trajectories are not realistic, they are surrealistic. (Englert et al. 1992, 1177-1178)

To sum up: in their opinion, their experiment clearly demonstrates that there is no sense to speak of "realistic" Bohmian trajectories. They conceded that Bohmian mechanics is at the present predictively equivalent to the standard non-relativistic quantum theory, and thus empirically correct, but claimed that Bohm's trajectory "can be macroscopically at variance with the detected, actual way through the interferometer", and thus "the interpretation of the Bohm trajectory-as the real retrodicted history of the atom observed on the screen-is implausible" (Englert et al. 1993, 1263); later, Scully et al. reaffirmed this view:

The Bohm trajectories are more metaphysical than physical.

[...] The reality attributed to Bohm trajectories is not physical, it is metaphysical.

(Scully et. al. 1998, 42, 45)

In the general debate following this experiment (Dürr et al. 1993; Englert et al. 1993; Dewdney et al. 1993; Scully et al. 1998; Griffiths 1999; Barret 2000; Hiley et al. 2000a; Hiley and Maroney 2000b), the main criticisms have been focused upon two points: the contextuality of all measurements and the inadequate use of the term "travelled path", as the standard view proscribes it in the absence of an actual meas- 
urement. Dewdney et al. presented (1993) a simpler version of such an experiment, without involving electromagnetic fields; they argued that "it reveals nothing in any way surrealistic about the Bohmian trajectories but rather illustrates in a dramatic way the influence of the nonlocal quantum potential" (Dewdney et al. 1993, 1), "the detector appears to have seen the particle, even though it has not passed through it" (1993, 9), as Englert et al. claimed, because "a detector in a interferometer does not behave in the same way as an isolated detector" and thus "it can become excited even when the electron passes along the other arm of the interferometer" $(1993,10)$. Hiley et al. argued that the supposedly observed trajectories can in no sense be established as the detected, actual trajectories, except for an additional assumption to standard theory: "that energy exchange only takes place when the actual atom interacts locally with the photon field in the [micromaser] cavity", this is, "if and only if the atom is physically present there" (Hiley et al. 2000a, 7). Moreover, they also state that "the storage of a single quantum of energy in the cavity does not constitute a measurement. There is no 'irreversible amplification"' (2000a, 9); therefore, the cavity is not considered to be a conventional position-measurement device for the position of the atom once this has passed through the interference region.

The last assertion makes clear that Bohmians finally accepted that there are situations where a delayed measurement by a reliable—-though non-conventional-whichway detector ends with the detector pointing along one path and the Bohmian particle travelling the other; of course they also stated that Bohm's theory predicts these facts properly, as the record by the detector is non-locally generated.

The last statement confronts us to what many consider the biggest problem of Bohmian theory: it allows operative non-locality, which is highlighted in experimental situations such as those described". In an additional example, Ahanorov et al. postulated a "protective measurement" (Ahanorov et al. 1993) on a particle in a box, in which the duration of a very weak interaction was large on all relevant time scales, therefore, and taking advantage of the characteristic adiabaticity of the temporal evolution, the wave function is protected against appreciable changes during the measurement. They found (Ahanorov et al. 1999) that the vast majority of Bohm's trajectories never came close to the box center where the interaction happens, that is to say that "the particle participates in a local interaction although its Bohm's trajectory", the succession of actual positions which are ascribed to the particle in Bohmian mechanics, "never comes near the interaction region" $(1999,137)$.

Drezet (2006) tried to answer their argument by denying that the process of interaction exposed by Ahanorov et al. would have involved a position measurement, but a measure of the density $|\psi(x \approx 0)|^{2}$, as the complete analysis of the interaction between two bodies requires the presence of the guiding wave. This answer, in my opinion, only reaffirms something nobody denies: Bohmian mechanics is consistent. But, all of these experiments ultimately reveal the fundamental incompatibility between Bohm's theory and relativity, as Barret pointed out:

${ }^{9}$ For a detailed analysis of how, and under what conditions, Bohmian mechanics allows the non-local transfer of a quantum of energy, see Solé $(2009,185 \mathrm{ff})$. 
Rather than saying that a detector is fooled by a late measurement, one should, I suggest, say that the late measurement reliably detects the position of the test particle nonlocally. (Barret 2000, 698)

To offer yet one more dramatic example of this non-locality, Hardy and Vaidman (Hardy 1992; Vaidman 2005), in order to remark that an empty wave-one without the Bohmian particle inside-can produce observable effects, considered a gedanken experiment in which a bullet splits its quantum wave at a beam-splitter into two equalweight wave packets, one moving towards a cat and another missing the cat, and concluded that we must also be worried about the cat's health ${ }^{10}$ when the Bohmian position of the bullet is inside the wave packet that misses the cat.

\section{Discussion}

Before starting my discussion, I want to advertise that an agreement seems to have been reached on that a proponent of Bohm's theory should not necessarily worry about this question, at least not if his main concern is that the theory continues to be considered empirically correct, as in this interpretation the wave function is taken as a wave field that cannot be dissociated from the particle and leads to the well-known main feature of Bohmian mechanics: the non-locality of phenomena. Once this is accepted, Bohm's particles need not follow the trajectories that one would expect from a local model, the core of most criticisms. But, the situations exposed must, in my opinion, disturb anybody who wants to establish Bohmian mechanics as a conventionally realistic theory.

To express this more clearly, let us consider the following situation: suppose that, as was first discussed by Englert et al. $(1992,1993)$ and after by Aharonov and Vaidman (Aharonov and Vaidman 1995; Vaidman 2005), the former cavity is replaced with a slow or "delayed" bubble chamber, and the atoms with particles that can ionize the liquid molecules in it. In the chamber, a particle could leave a track that is produced by a succession of local interactions and it does not coincide with the corresponding Bohm's trajectory:

It is even more dramatic when our particle moves inside a special bubble chamber. The bubbles created due to the passage of the particle are developed slowly enough such during the time of the motion of the particle the density of the spatial wave function of each bubble does not change significantly. Then, what we will see as a trace of bubbles is the particle moving from the left to the right, while the Bohmian particle, in fact, will move from the right side, stop, and come back to the right. (Aharonov and Vaidman 1995, 11)

The result of the experiment (which can be seen only much later) is a trace of bubbles corresponding to one trajectory while the trajectory of the Bohmian position is the other one. The bubbles show the trajectory of the empty wave! (Vaidman 2005, 305)

A divergence appears between two representations, the Bohmian (trajectories) and the-almost inevitable in its construction-mental human representation corresponding to what we observe or "see" at the macroscopic level (trace of bubbles), that is delayed (the bubbles appear slowly) with respect to the sequence of events that allegedly would be observed in real time:

10 To tell the truth, instead of killing a cat, the bullet flips spins on its way, along a different trajectory to the Bohmian trajectory. 
Note, however, that the picture we see via appearance of the bubbles is time delayed. In a "fast" bubble chamber, in which we see a real time motion, the Bohmian particle moves together with the trace of bubbles. (Aharonov and Vaidman 1995, 11-12)

Thus, we face a paradoxical situation: the development of an alternative theory to the orthodox, with realistic pretension and including pretended objective trajectories, has given rise to an interpretation that shows, for certain phenomena, a radical mutual incompatibility with the classical narration (based on classical trajectories and local interactions) by means of which these phenomena, once finalized, are narrated or communicated in the standard interpretation.

What (Bohmian) descriptive mental model can we develop, then? Perhaps an alternative, fully consistent empirically, according to which, in some sense, it would be possible to say that the wave function is observed, as it is its empty part the one that makes the detectors jump. Whereas, the particle is supposedly occupying an objective position that is not susceptible to direct observation in real time, but it is determined retrodictedly once the experiment has finalized, since when the experiment is finished the measured position of the particle is not the formerly occupied, as Barret pointed out:

An observation that one might have thought would (locally) determine where a test particle was early in an experiment might instead (nonlocally) determine where it $i s$ at the moment the experiment is completed. (Barret 2000, 702)

Thus, for the bubble chamber described and despite the wake of bubbles, the Bohmian experimenter has to narrate the particle travelled elsewhere, other than the path indicated by the bubbles: along a path relativistically separated from the zone of its formation. In stark contrast, although it is true that the orthodox physicist would be fully aware he has not observed a trajectory, at least he could elaborate a descriptive model of the experience in which it is narrated as if the particle had followed a path locally connected with the trace of bubbles. This model is always constructed a posteriori in an experiment with delayed election, and it is thought out just because of finding it useful to communicate the achieved results.

In any case, the orthodox quantum experimenter is fully aware that his narrative model does not imply that, if he had measured at the earlier time, he would have determined the path assigned in the classic model of visualization. For this reason, as has been repeatedly warned, the use of these kinds of narratives could lead to confusion and are increasingly considered as superfluous. The main justification for the persistence of such models would be, after all, the fundamental assumption that the theory only can admit local interactions: a detector cannot be shot, in any case, by a remote faster than light signal; that is to say, if the detector is triggered it is because of the particle being inside its local surroundings.

Note that, while the orthodox narrative only has a communicative purpose, and must be understood like a mere narrative resource, without any aspiration to describe what really has happened, Bohmian narrative, on the contrary, pretends to be the real description of what happened: The particle moved occupying successively the positions that conform to the corresponding trajectory and the detector jumped by a nonlocal effect. 
Consequently, with respect to the Bohmian interpretation:

1. The Bohmian trajectories, despite their assumed realistic character, appear as just mathematical constructs with no relation to the common concept of trajectory based on local measures of positions, since usual position measurements, which rely on local interactions to determine the actual position of the particles, cannot measure (locally) the supposedly objective Bohmian position. Thus, Bohm position emerges like a variable that it is not possible to measure by means of standard position detectors, and "the concept of position itself becomes shaky" (Ahanorov et al. 1999, 137).

2. If the Bohmian trajectories are revealed as metaphysical, the wave function, which is in general a function on $3 \mathrm{~N}$-dimensional configuration space, becomes observable, in the following sense: an empty wave-which does not contain the Bohmian particle inside_can produce observable effects, like triggering a detector.

3. The situation is so peculiar that the non-Bohmian could doubt, once absolute priority to the requirement of locality for all interactions is given, if it makes sense to continue calling "position" the corresponding Bohmian hidden variable. Alternatively, it could be proposed to call some "orthodox positions" detectors as "Bohmian's wave field" detectors.

And then, it could perhaps be said that the wave function emerges, in some sense, as real, despite such an affirmation appearing to be, at least, problematic. But, this was the view of Bohm himself and it is probably the majority opinion among Bohmians; however, other authors have adopted different ontosemantic commitments, from monism to dualism. Some researchers have postulated that only particles are entities in real physical space whilst others maintain that both particles and field-waves are real physical entities. Whatever, it is worth bearing in mind Bell's statement that "no one can understand this theory until he is willing to think of $\Psi$ as a real objective field rather than just a 'probability amplitude'. Even though it propagates not in 3-space but in 3N-space" (Bell 1981, 618; Dürr et al. 1997). A general discussion of the different ontologies associated to Bohmian formalism is to be found in Belousek (2003).

4. A fundamental question arises: what is the meaning of the term "realistic" when applied to the Bohm's theory? Formerly, I have apparently conflated the concepts of "real" and "susceptible of direct observation", the latter conceived as "able to trigger a given experimental set-up". But how the term "real" can be understood is a complex and old topic that I, obviously, do not pretend to discuss, merely stating here two senses of the term that are completely inadequate for the Bohmian position and trajectories:

a) If "real" is granted to mean simply "classical", it is obvious that neither the Bohmian particle nor its position are realistic, as it is well-known that, as we have said before, Bohmian mechanics does not represent a return to the classical paradigm but a radical challenge to it: Bohmian mechanics is a new, distinctly non-Newtonian mechanics ${ }^{6-7}$. For instance, it turns out that, be- 
cause of the effects of the wave function on the evolution of its position, the trajectory of a single particle in free space is not necessarily a simple straight line, and an electron in the ground state of a hydrogen atom is motionless.

b) It is also possible to speak of "real" with the meaning of "objective", that is, to consider the Bohmian positions as realistic just because of their objectivity: the theory assumes that each particle occupies a position whether being observed or not. But, then the problem of the surrealistic trajectories shows that this is a hypothesis that cannot be tested experimentally, as our actual positions detectors can be affected non-locally and, therefore, the Bohmian actual position remains ineludibly as a hidden variable.

5. There are phenomena whose description, contrary to the situation in the standard interpretation, is fully incompatible with the development of any mental model akin to a classical description in which, illustratively and according to the model suggested by macroscopic facts, we visualize the particle moving along a path marked by the detectors. For instance, in the formerly referred bubble chamber, the generated bubbles do not enclose the Bohmian particle.

We can remember now the following words by Einstein, in relation with the fact that, for a one-dimensional Bohmian particle in a box with purely elastic sides, its velocity is zero at all times:

The vanishing of the velocity contradicts the well-founded requirement that in the case of a macroscopic system, the motion must approximately agree with the classical mechanical motion. (Einstein 1953, 5)

In sharp contrast, the Bohmian interpretation banishes any possibility of coexistence between the unobserved (but supposedly objective) trajectory of the Bohm particle and the classical intuitive picture inferred from macroscopic (local) observations.

6. An operative non-locality of the theory is confirmed, that makes it fundamentally incompatible with relativity and that, beyond any onto-epistemological discussion, can be considered like the defect of the theory. Accepting the non-local transference of a quantum of energy seems to go too much far (at least at present times).

\section{Final remarks}

The previous experimental contexts make it clear that it is highly problematic to state that Bohm's trajectories are "realistic", at least in the most common senses of the term. Besides, none of the previous results establishes Bohmian mechanics as inconsistent; what they reveal is that Bohm's trajectories cannot be linked in any way, neither to the classical ones nor to the positions measured by our usual positions detectors. And, more dramatically, they show that there is a fundamental incompatibility between Bohm's theory and relativity (see e.g. Barrett 2000 and Solé 2009).

The experimental contexts described support the idea that Bohmian position is unobservable and at odds with the usual understanding of the term, based on the con- 
cept of local interaction and - like the orthodox quantum variable - assumed as susceptible to measurement by the corresponding detectors. And it happens even in experimental situations, like the formerly mentioned bubble chamber, in which the orthodox interpretation can be concealed with a "classical" narrative elaborated only to describe the observed phenomena in a way that agrees with our usual macroscopic understanding - although it is not assumed as a "real" narration of what has happened.

To sum up, if we adopt either the orthodox formulation of quantum mechanics or the Bohm alternative, we are compelled to accept a radical challenge to previous classical terminology and, more fundamentally, to classical ontology. Thus, we are compelled to ask a fundamental question: Why should we insist on considering as "realistic" a metaphysics which, while seeming to express the essential ontological commitment missing from orthodox quantum theory is, in fact, strongly at odds with the semantics we use to communicate our experiments when a classical narrative model is plausible?

The final (and open) question is thus: in the end, in which sense can we accept the Bohm's positions and trajectories as "realistic"? I believe that the proper answer from Bohmian's to the problem of surrealistic trajectories must include a reconsideration of the meaning in which Bohmian theory can continue being named as "realistic".

And, if both orthodox and Bohmian quantum mechanical interpretations are accepted as unrealistic, it would seem that a fundamental issue is reinforced: that experimentation could have led us to know a world whose phenomena turn out to be describable by means of logical and mathematical structures and concepts existing only in our minds or, at least, not connected to properties experimentally accessible in their objectivity, in case they owned any. But we do not have to accept this as the final and definitive situation; in future, other theory might come to restore the realism in science, by introducing new concepts, outside the classical but liable to be called "realistic". As d'Espagnat wrote:

The idea according to which any macroscopic object necessarily occupies some definite region of space- to the exclusion of all other regions-is not an obvious (and hence unquestionable) truth, but rather an element of the definition, useful in given circumstances, of the word "object". Correlatively, it also gives us hope that, even if our atomistic descriptions-or our descriptions in terms of "objects"- of basic reality ultimately turn out to be inadequate, this is no reason for us to abandon the attempt to describe said reality, and to become "die-hard positivists". Perhaps other concepts will emerge which will be better fitted for this purpose. (Espagnat 1976, xx-xxi)

Finally, I raise an old and fundamental question that probably never will find a definitive answer: whether any kind of realism can survive, as science must try to describe an assumed reality underlying but remote from phenomena; or whether, on the contrary, "such speculations seem to us, to say it explicitly, fruitless and senseless. Physics ought to describe only the correlation of observations" (Heisenberg 1983, 83). In any case, "the probing of what quantum mechanics means must continue, and in fact it will continue, whether we agree or not that it is worthwhile, because many people are sufficiently fascinated and perturbed by this that it will go on" (Bell 1986, 52). 


\section{REFERENCES}

Aharonov, Y., J. Anandan, and L. Vaidman. 1993. Meaning of the wave function. Physical Review A47, $\mathrm{n}^{\circ}$ 6: 4616-4626.

Aharonov, Y. and L. Vaidman. 1995. About position measurements which do not show the Bohmian particle position. ArXiv.org, http://arxiv.org/abs/quant-ph/9511005v1 (accessed December 11, 2011). In Bohmian Mechanics and Quantum Theory: An Appraisal, ed. J.T. Cushing, A. Fine and S. Goldstein. Boston Studies in the Philosopby of Science 184: 141-154. Dordrecht: Springer.

Ahanorov, Y., B-G Englert and M. O. Scully. 1999. Protective measurements and Bohm trajectories. Physics Letters A263: 137-146.

Barrett, J.A. 2000. The persistance of memory: Surreal Trajectories in Bohm's Theory. Philosophy of Science 67: 680-703.

Bell, J.S. 1964. On the Einstein-Podolsky-Rosen paradox. Physics 1: 195-200.

- 1980. De Broglie-Bohm, Delayed choice double slit experiment, and density matrix. International Journal of Quantum Chemistry: Quantum Chemistry Symposium 14: 155-159.

- 1981. Quantum Mechanics for Cosmologists. In Quantum Gravity 2, ed. C. Isham, R. Penrose and D. Sciama, 611-637. Oxford: Clarendon Press.

Bell, J.S. 1986. In The ghost in the atom, ed. P. C. W. Davies and J. R. Brown, 45-57. Cambridge: Cambridge University Press.

Belousek, D.W. 2003. Formalism, Ontology and Methodology in Bohmian Mechanics. Foundations of Science 8: 109-172.

Bohm, D. 1952. A suggested interpretation of the quantum theory in terms of 'hidden' variables. I and II. Physical Review 85: 166-193.

Bohm, D., B. J. Hiley and P. N. Kaloyerou. 1987. An ontological basis for the quantum theory. Physics Reports 144: 321-375.

Bohm, D. and B. J. Hiley. 1993. The Undivided Universe. An Ontological Interpretation of Quantum Theory. London: Routledge.

Bohr, N. 1934. Atomic theory and the description of nature. Cambridge: Cambridge University Press.

Broglie, L. de. 1927. La Mècanique Ondulatoire et la structure atomique de la matière et du rayonment. Le Journal de Physique et Le Radium 8: 225-241.

Daumer, M., D. Dürr, S. Goldstein and N. Zanguí. 1996. Naive realism about operators. Erkenntnis 45 , n $^{\circ}$ 2-3: 379-397.

Dewdney, C., L. Hardy and E. J. Squires. 1993. How late measurements of quantum trajectories can fool a detector. Physics Letters A184: 6-11.

Drezet, A. 2006. Comment on 'Protective measurements and Bohm trajectories'. Physics Letters A350: 416418.

Dürr, D., S. Goldstein and N. Zanguí. 1992a. Quantum Equilibrium and the Origin of Absolute Uncertainty. Journal of Statistical Physics 67: 843-907.

—. 1992b. Quantum mechanics, randomness and deterministic reality. Physics Letters A172: 6-12.

Dürr, D., W. Fusseder, S. Goldstein and N. Zanguí. 1993. Comment on 'Surrealistic Bohm Trajectories'. Zeitschrift für Naturforschung 48a: 1261-1262.

Dürr, D., S. Goldstein and N. Zanguí. 1996. Bohmian Mechanics as the Foundation of Quantum Mechanics. In Bohmian Mechanics and Quantum Theory: An Appraisal, ed. J.T. Cushing, A. Fine and S. Goldstein. Boston Studies in the Philosophy of Science 184: 21-44. Dordrecht: Springer.

- 1997. Bohmian Mechanics and the Meaning of the Wave Function. In Experimental Metaphysic-Quantum Mechanical Studies for Abner Shimony. Vol. 1, ed. R. S. Cohen, M. Horne and J. J. Stachel. Boston Studies in the Philosophy of Science 193: 25-38. Dordrecht: Springer.

Einstein, A., B. Podolsky and N. Rosen. 1935. Can Quantum-Mechanical description of physical reality be considered complete?. Physical Review 47: 777-780.

Einstein, A. 1953. Elementary Considerations on the Interpretation of the Foundations of Quantum Mechanics, transl. D. Karanth, 2011. ArXiv.org.

http://arxiv.org/PS_cache/arxiv/pdf/1107/1107.3701v1.pdf (accessed December 11, 2011). Originally published in Scientific Papers Presented to Max Born On His Retirement From The Tait Chair of Natural Philosophy In The University of Edinburgh, 33-40. New York: Hafner Publishing Company. 
Englert, B.-G., M. O. Scully, G. Süssmann and H. Walther. 1992. Surrealistic Bohm trajectories. Zeitschrift für Naturforschung 47a: 1175-1186.

—. 1993. Reply to "Comment on 'Surrealistic Bohm trajectories' ". Zeitschrift für Naturforschung 48a: 12631264

Espagnat, B. d’. 1976. Conceptual Foundations of Quantum Mechanics. Massachusetts: Benjamin.

Goldstein, S. 1996. Bohmian Mechanics and the Quantum Revolution. Synthese 107: 145-165.

Griffiths, R.B. 1999. Bohmian mechanics and consistent histories. Physics Letters A261: 227-234.

Hardy, C. 1992. On the existence of empty waves in quantum theory. Physics Letters A167: 11-16.

Heisenberg, W. 1983. The physical content of quantum kinematics and mechanics. In Quantum Theory and Measurement, ed. J. A. Wheeler and W. H. Zurek, 62-84. Princeton: Princeton University Press. Originally published in Zeitschrift für Physik 43: 172-198, 1927.

-. 1985. Reminiscences from 1926 and 1927. In Niels Bobr. A Centenary Volume, ed. A. P. French and P. J. Kennedy, 163-171. Harvard: Harvard University Press.

Hiley, B. J., R. E. Callaghan and O. J. E. Maroney. 2000a. Quantum trajectories, real, surreal or an approximation to a deeper process?. ArXiv.org, http://arxiv.org/abs/quant-ph/0010020v2 (accessed December 11, 2011).

Hiley, B. J. and O. J. E. Maroney. 2000b. Consistent histories and the Bohm approach. ArXiv.org. http://arxiv.org/abs/quant-ph/0009056v1 (accessed December 11, 2011).

Jacques, V., E. Wu, F. Grosshans, F. Treussart, P. Grangier, A. Aspect and J.-F. Roch. 2007. Experimental Realization of Wheeler's Delayed-Choice Gedanken Experiment. Science 315, no 5814: 966968.

Jönsson, C. 1961. Elektroneninterferenzen an mehreren künstlich hergestellten Feinspalten. Zeitschrift für Physik 161, nº 4: 454-474.

Kennedy, P. J. 1985. Delayed-Choice Experiments. In Niels Bobr. A Centenary Volume, ed. A. P. French and P. J. Kennedy, 148-152. Harvard: Harvard University Press.

Merli, P. G., G. F. Missiroli and G. Pozzi. 1976. On the statistical aspect of electron interference phenomena. American Journal of Physics 44: 306-307.

Myrvold, W.C. (2003). On some early objections to Bohm's theory. International Studies in the Philosophy of Science $17, \mathrm{n}^{\circ}$ 1: 7-24.

Petersen, A. 1985. The Philosophy of Niels Bohr. In Niels Bohr. A Centenary Volume, ed. A. P. French and P. J. Kennedy, 299-310. Harvard: Harvard University Press.

Philippidis, C., C. Dewdney and B. J. Hiley. 1979. Quantum interference and the Quantum Potential. Il Nuovo Cimento 52B: 15-28.

Scully, M.O., B.-G. Englert and H. Walther. 1998. Do Bohm trajectories always provide a trustworthy physical picture of particle motion?. Physics Scripta T76: 41-46.

Solé Bellet, A. 2009. Realismo e interpretación en Mecánica Bohmiana. PhD diss., Universidad Complutense de Madrid.

Tonomura, A., J. Endo, T. Matsuda and T. Kawasaki. 1989. Demonstration of single-electron buildup of an interference pattern. American Journal of Physics 57, $\mathrm{n}^{\circ}$ 2: 117-120.

Vaidman, L. 2005. The Reality in Bohmian Quantum Mechanics or Can You Kill with an Empty Wave Bullet?. Foundations of Physics 35, $\mathrm{n}^{\circ}$ 2: 299-312.

Wheeler, J.A. 1978. The 'Past' and the 'Delayed-Choice' Double-Slit Experiment. In Mathematical Foundations of Quantum Theory, ed. A. R. Marlow, 9-48. New York: Academic Press.

- 1983. Law without law. In Quantum Theory and Measurement, ed. J. A. Wheeler and W. H. Zurek, 182213. Princeton: Princeton University Press.

Zeilinger, A. 1999. Experiment and the foundations of quantum physics. Reviens of Modern Physics 71, $\mathrm{n}^{\circ} 2$ S288-S297.

Zetie, K. P., S. F. Adams and R. M. Tocknell. 2000. How does a Mach-Zehnder interferometer work?. Physics Education 35, $\mathrm{n}^{\circ}$ 1: 46-48.

MARÍA C. Boscá is senior lecturer in Atomic, Nuclear and Molecular Physics at the University of Granada (Spain). She has published on nuclear physics, particularly on many-body theories, and has recently started working on general issues concerning the philosophy of quantum mechanics.

AddRess: Departamento de Física Atómica, Molecular y Nuclear, Universidad de Granada, Campus Fuentenueva, E-18071 Granada, Spain. E-mail: bosca@ugr.es 